\title{
Comparison of different image intensifiers used for combustion measurement
}

\author{
Jan Kouba ${ }^{1,2, *}$, Jan Novotný ${ }^{3}$, and Martin Mulenko ${ }^{4}$ \\ ${ }^{1} \mathrm{CTU}$ in Prague, FME, Department of Automotive, Combustion Engine and Railway Engineering, 16607 Prague, Czech Republic \\ ${ }^{2} \mathrm{CTU}$ in Prague, FME, Department of Fluid Mechanics and Thermodynamics, 16607 Prague, Czech Republic \\ ${ }^{3}$ J. E. Purkyně University in Ústí nad Labem, FME, Department of Machines and Mechanics, 40001 Ústí nad Labem, Czech Republic \\ ${ }^{4}$ CTU in Prague, FME, Department of Process Engineering, 16607 Prague, Czech Republic
}

\begin{abstract}
This paper deals with setting of the acquisition equipment used to visualize and measure the concentration of intermediate species chemiluminescence combustion and laser-induced fluorescence. Observed intermediates such as $\mathrm{OH}, \mathrm{NO}, \mathrm{CH}$ and others have an emitted $\mathrm{UV}$ spectrum and their signal is very weak. For this reason, it is necessary to use a suitable image intensifier, and its proper setting and synchronization with the camera is also very important. In view of this problem, the following text deals with the appropriate setting of selected optical amplifiers with P46 and P43. Because the recording equipment can be used for both LIF and chemiluminescence, the experimentally determined setting will be performed using the $\mathrm{OH}$-chemiluminescence method. This is reported by a weaker signal and creates a more stringent setup criterion. As a qualitative factor, a gradient of the image function has been selected, which plays a significant role in edge detection and therefore also in flame front position detection.
\end{abstract}

\section{Introduction}

Laser Induced Fluorescence (LIF) and Chemiluminescence are methods frequently used in the visualization and measurement of the combustion processes. Using these methods, it is possible to monitor the spatial distribution of individual intermediate species and thus to contribute to better understanding of processes involved in combustion. Their advantages consist mainly in the non-invasive character with high spatial resolution. The chemiluminescence method is an easier method designed mainly for visualization, as it observes a whole envelope of monitored intermediate species. It is then recorded as a projection into the image. In contrast, the LIF method is capable of detecting selected intermediate species in a plane intersected by laser sheet, which is its integral part. Both advantages and disadvantages come from the method above. Advantages include, for example, a possibility of quantitative measurement of both concentrations and temperature in a preselected plane. This allows LIF to uncover the flame structures, which are partly hidden for the chemiluminescence method. Disadvantages of LIF include especially lengthy and difficult laser setting or calibration connected with very complicated quantitative measurement [3]. $\mathrm{CH}, \mathrm{NO}$ or $\mathrm{OH}$ radicals [1] belong among frequently visualized ones. The principle of visualization of both methods consists in recording the luminescence of selected intermediate species. Both methods make use of the same recording equipment. It is therefore possible to apply the LIF setting to chemiluminescence method and vice versa when searching for optimal setting of the recording equipment and selection of an image intensifier. This eliminates the manipulation with tuneable dye laser used for LIF method and thus the lengthy setting of the wavelength suitable for excitation of the selected intermediate species. The recorded luminescence of the intermediate species is very weak, very often located in the UV area; its signal passes through the band-pass filter, in case of monitoring the $\mathrm{OH}$ radicals with a throughput of approximately $40 \%$. For these reasons, it is necessary to use the image intensifier that amplifies the signal and allows for its recording for the CCD camera. The intensity of the detected signal can be influenced in several ways. In case of LIF, an increase in the energy of the excitation beam can be made use of; energy of the excitation beam can be increased either by simple increase in power level of the pulse Nd-YAG laser or by better adjustment of the whole optical system. Another way of increasing the intensity of the detected signal is to use more sensitive camera with a more powerful image intensifier. Last but not least, it is possible to increase the intensity of the detected signal by suitable synchronization of the whole recording equipment. Setting and synchronization of the recording equipment, including the image intensifier, provides the ability to influence the quality of the recorded data. Sharp and sufficiently intense detected luminescence signal emitted by monitored intermediate species is crucial for quality LIF measurement or visualization with the help of

Corresponding author: Jan.Kouba@,fs.cvut.cz 
chemiluminescence. This work deals with an impact of synchronization and length of CCD exposure on the resulting intensity of the recorded signal for both methods. Given the easier manipulation during data acquisition, the method of chemiluminescence of $\mathrm{OH}$ radicals was chosen.

\section{Experimental setup}

The experimental setup consists of a rigid frame, which includes propane-butane lamp serving as a signal source. Attached to the frame is a pivoting arm with the recording equipment. The equipment is controlled by DynamicStudio software, which also allows for data recording and processing. The scheme of the experiment and its arrangement can be seen in Fig. 1 and Fig. 2.

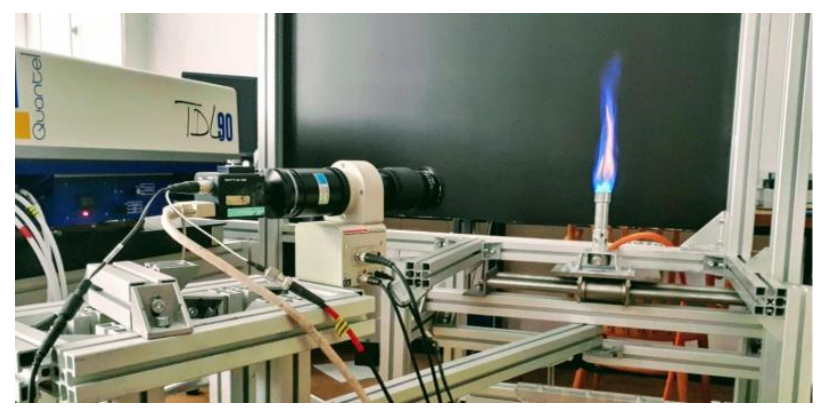

Fig. 1. Experiment arrangement.

The main components of the recording equipment are the following:

- Band-pass filter

- Quartz glass objective

- Image intensifier

- Objective between the CCD and the image intensifier

- CCD camera

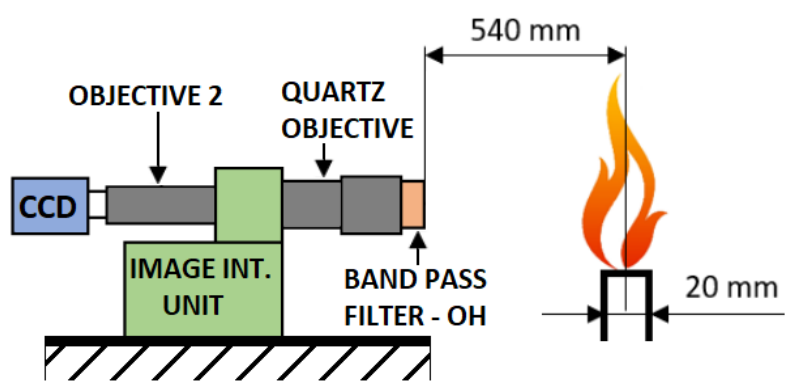

Fig. 2. Acquisition equipment set-up.

In order to obtain data for comparing the image intensifiers, the $\mathrm{OH}-\mathrm{Chemiluminescence} \mathrm{method} \mathrm{was}$ used. $\mathrm{OH}$ radicals originating at the flame front and emitting a wavelength signal of about $310 \mathrm{~nm}$, i.e. in the UV range. For the reason above, it is necessary to use special input Quartz glass optics allowing the passage of UV radiation. The optics includes a band-pass filter with a maximum throughput at a wavelength of $313 \mathrm{~nm}$ and a bandwidth of $25 \mathrm{~nm}$. A standard FlowSense 4M MKII CCD camera designed for PIV, but also suitable for LIF and chemiluminescence, was used. The basic parameters of this camera is resolution of $2048 \times 2048$ pixels with a bit depth of 12 bit. A pair of image intensifiers made by Hamamatsu company was used. These are C9547-03 type and C10880-03 type. The image intensifiers are mostly devices using photoelectric emission, luminescence and sometimes secondary electron emission. The basic elements of the image intensifier are also based on these phenomena. This is a photo cathode ensuring the electron emission when absorbing the radiation. On the opposite side there is a luminescent screen. Between these two elements, an electron accelerating field is being created. [4] Accelerating electrons hit the screen and luminescence occurs. The luminescent screen has time required for dimming, depending on the material used. [7] The maximum repetition rate of the intensifier depends on the length of time necessary for dimming. The repetition rate affects usability of the intensifier for individual applications and, last but not least, the quality of the data. A micro channel plate (MCP) using secondary electron emission is placed between the photo cathode and luminescent screen. MCP significantly increases the number of electrons hitting the screen, and thus the profitability of the image intensifier. Compared intensifiers have similar design, except that a different type of luminophore is used; P43 for C9547-03 type and P46 for C10880-03 type. Other parameters of the intensifiers are listed in the following Table 1., which confirms the similarity of image intensifiers input. The Fig. 3 shows evolution of the relative intensity of the intensifier's output signal depending on time for different gating times. The figure shows a big difference in the decay time of individual luminophores. However, the manufacturer does not provide information about the course of the absolute value, and it is very difficult to determine, from the data available, which of the image intensifiers provides better quality data for measurement or visualization with the help of the LIF and Chemiluminescence method.

Table 1. Image intensifiers parameters.

\begin{tabular}{|c|c|c|}
\hline Type & C 9547-03 & C10880-03 \\
\hline $\begin{array}{c}\text { Luminous } \\
\text { sensitivity } \\
{[\mu \mathrm{A} / \mathrm{lm}]}\end{array}$ & 230 & 230 \\
\hline $\begin{array}{c}\text { Radiant sensitivity } \\
{[\mathrm{mA} / \mathrm{W}]}\end{array}$ & 53 & 53 \\
\hline $\begin{array}{c}\text { Quantum } \\
\text { efficiency [\%] }\end{array}$ & 15 & $\mathrm{P} 46$ \\
\hline $\begin{array}{c}\text { Phosphor screen } \\
\text { Luminous gain } \\
{[(\text { lm/m2)/lx] }}\end{array}$ & $1 \cdot 10^{4}$ & $1 \cdot 10^{5}$ \\
\hline $\begin{array}{c}\text { Gate maximum } \\
\text { repetition } \\
\text { frequency }[\mathrm{kHz}]\end{array}$ & 30 & 200 \\
\hline
\end{tabular}




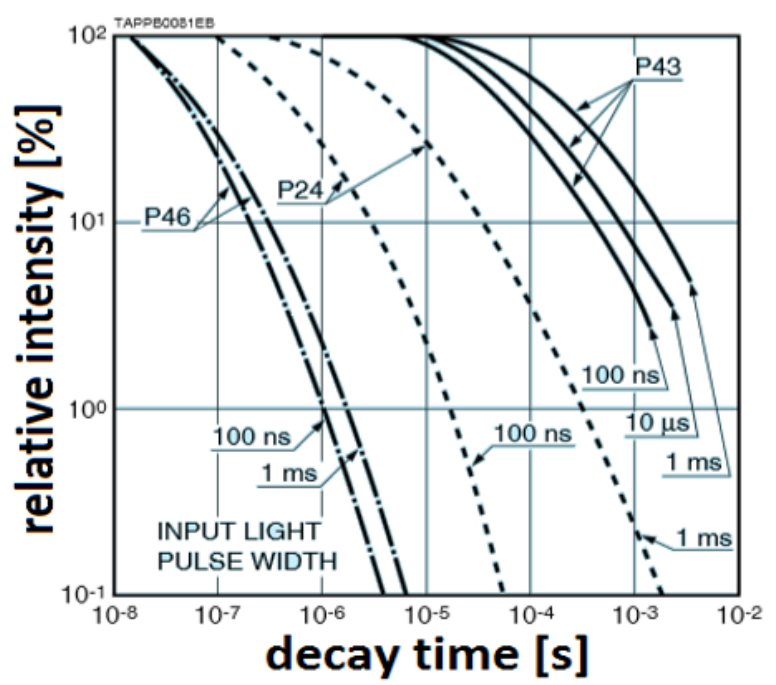

Fig. 3. Phosphor screen decay characteristics for different types of luminophores. [7]

Considering the above, it is apparent that appropriate setting of the image intensifier and camera leads to better results of the recorded data, for both methods.

\section{Processing and analysis of the measured data}

In order to evaluate the optimal setting of the assembly and subsequent selection of a more suitable intensifier, a pair of evaluation parameters was used, which are based on conventional data processing using the chemiluminescence method and laser-induced fluorescence. These criterions are ratio signal-to-noise and maximum value of gradient.

\subsection{Signal-to-noise ratio}

The whole tested equipment has a very high level of noise. It consists of a camera noise factor and image intensifier noise factor. The resulting noise level is for this comparison referred to as absolute value of mean function which is obtained by subtracting the measured data from data modified by median filter function. For data modification has been used local median smoothening defined by the relationship in eq. (1)[4].

$$
g(x, y)=\sum_{k=-N}^{N} \sum_{l=-N}^{N} h(k, l) f(x+k, y+l)
$$

The principle of this smoothening is, that to every point, there is a new value assigned which is median value in area $5 \times 5$ pixels. The value $x$ and $y$ are coordinates of an image of function $f, h$ is a median mask and $g$ is a resulting image function. One of the quality indicator of the recorded data is the ratio between the maximum value of the image function and the noise thus evaluated, according to the relation (2). Although the noise level of such assembly is relatively high. When using the same camera while taking images, its noise factor has no impact on the resulting selection of a more suitable image intensifier.

$$
S N R=\frac{\max (f(x, y))}{|\operatorname{mean}(g(x, y)-f(x, y))|}
$$

\subsection{Maximal gradient}

The aim of comparing the assembly is to find and optimal setting for using in Chemiluminescence methods and in laser-induced fluorescence methods. One area of application of these methods is the flame front detection. In order to find its position, we often use edge detection in the image with the help of maximum image function gradient value, which is defined by this relationship. (3) Therefore, the maximum image function gradient value is used as another quality indicator of the recorded data.

$$
\nabla f(x, y)=\sqrt{\left(\frac{\partial f}{\partial x}\right)^{2}+\left(\frac{\partial f}{\partial y}\right)^{2}}
$$

\subsection{Processes of evaluation}

Data was acquired by the commercial Dynamic Studio software, which allows both data capture and storage as well as synchronization settings for the recording equipment. For each mode, 100 images were taken. Every serie was to "tiff" format exported and imported to MATLAB for other evaluation.

\subsubsection{Maximal gradient evaluation process}

- Position of signal cross section selection

- Evaluation of the mean value in the cross section

- Searching the maximum gradient value

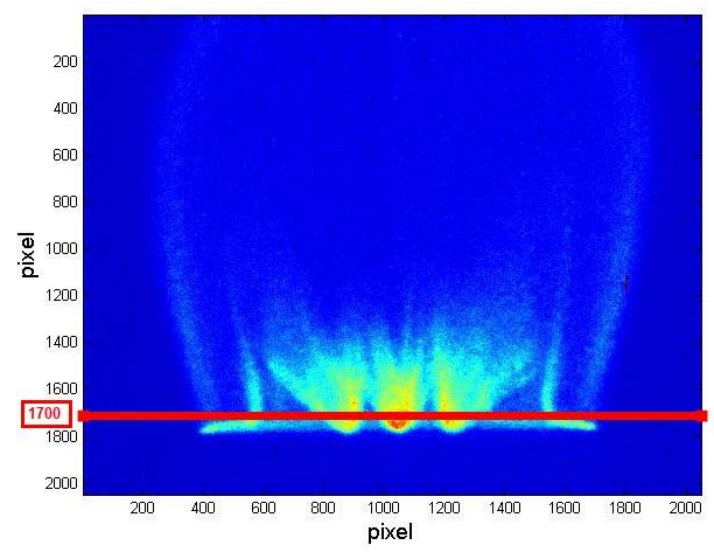

Fig. 4. Position of signal cross-section, highlighted by red line

Selection of the cross-sectional position was based on observation. The Fig. 4 shows that the position of the cross section could include maximal gradient values. The following pictures (Fig. 5-12) show the strongest and weakest images of both intensifiers. On the right side of each image is cross-sectional signal course of serie with median value. 


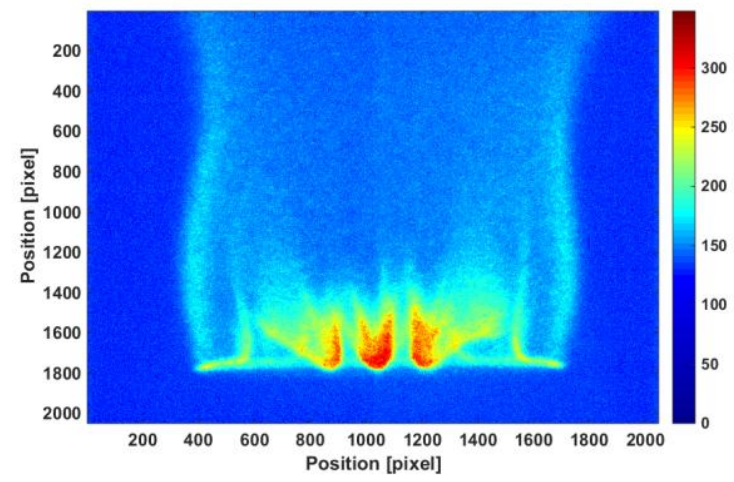

Fig. 5. C 9547-03, 25\% gain, exposur time $300 \mu \mathrm{s}$

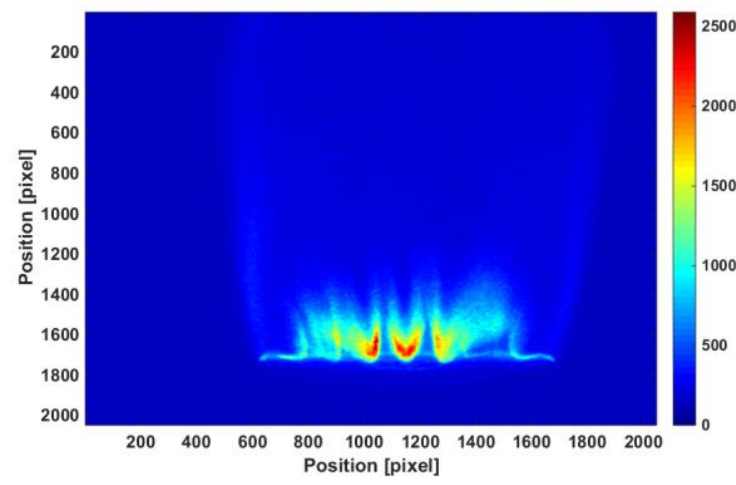

Fig. 7. C 9547-03, 75\% gain, exposure time $300 \mu \mathrm{s}$

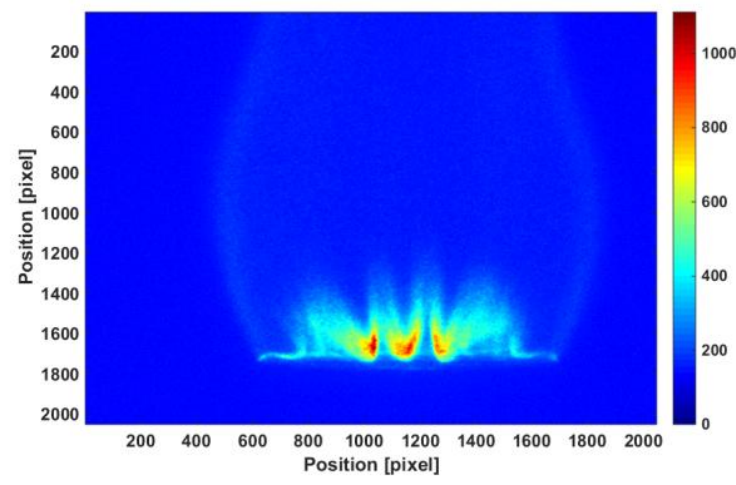

Fig. 9. C 10880-03, 25\% gain, exposure time $20 \mu \mathrm{s}$

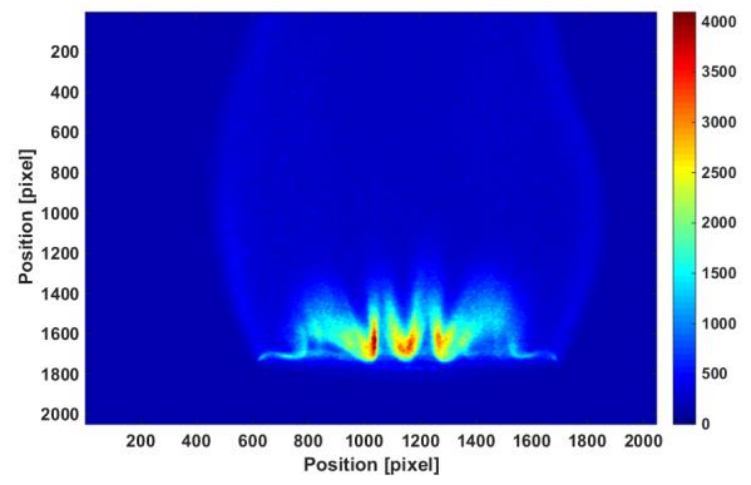

Fig. 11. C 10880-03, 60\% gain, exposure time $300 \mu \mathrm{s}$

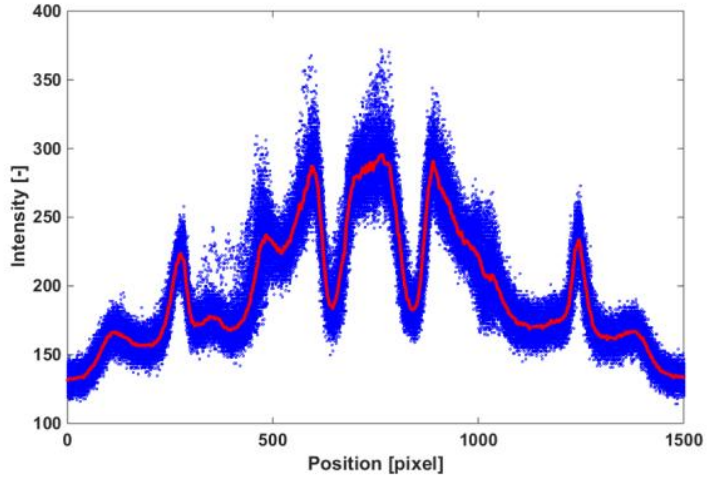

Fig. 6. C 9547-03, 25\% gain, exp. time $300 \mu$ s, cross-section

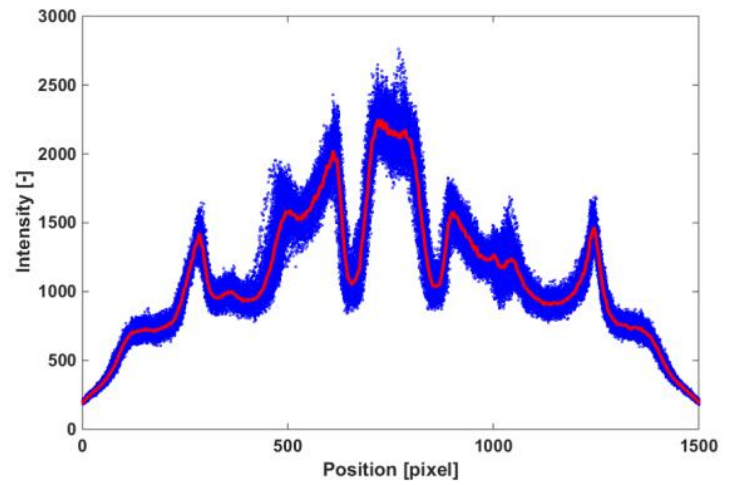

Fig. 8. C 9547-03, 75\% gain, exp. time $300 \mu$ s , cross-section

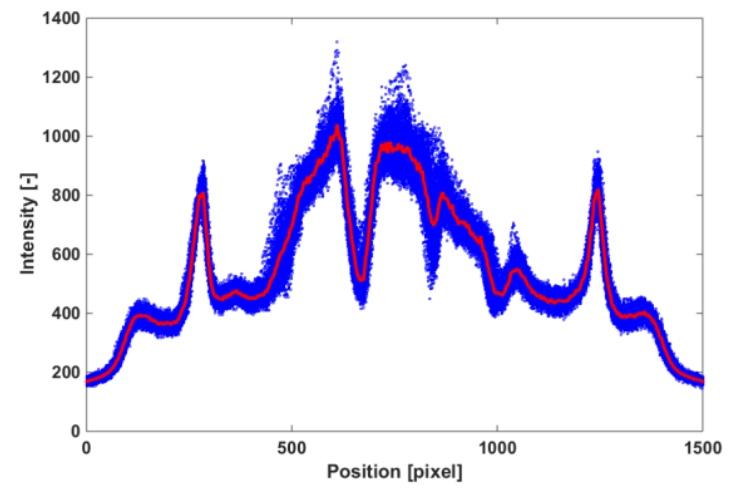

Fig. 10. C 10880-03, 25\% gain, exp. time $20 \mu$ s, cross-section

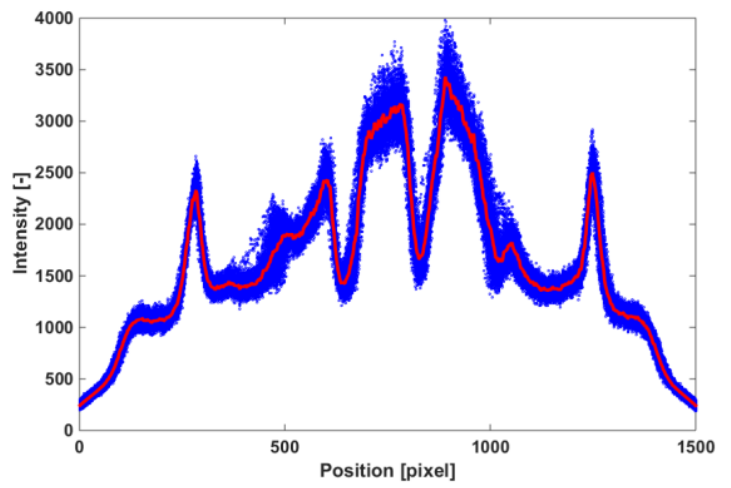

Fig. 12. C 10880-03, 60\% gain, exp. time $300 \mu$ s, cross-section 


\subsubsection{Signal-to-noise evaluation process}

- Median smoothing of each image

- Noise component obtaining by original and smoothed image subtraction

- Maximal value divided by noise component

The following pictures (Fig. 13, 14) show comparison between acquired and smoothed image.

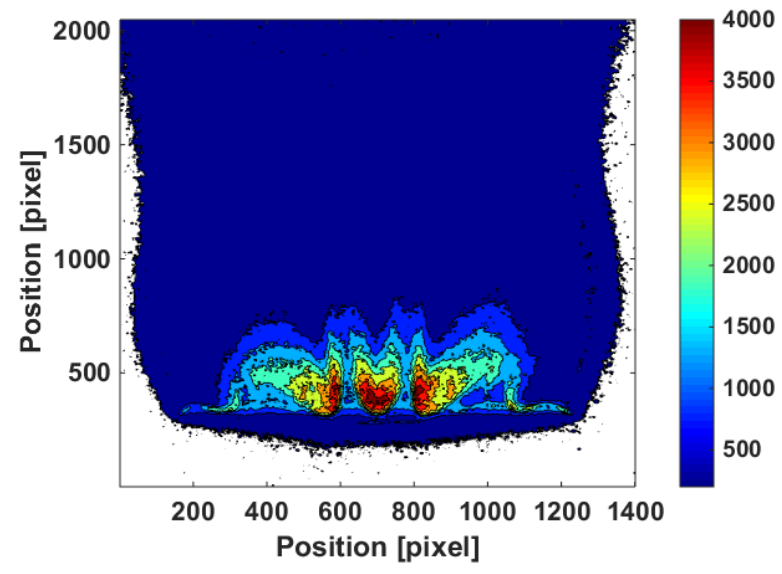

Fig. 13. C 10880-03, 60\% gain, exposure time $300 \mu$ s, contours of acquired image

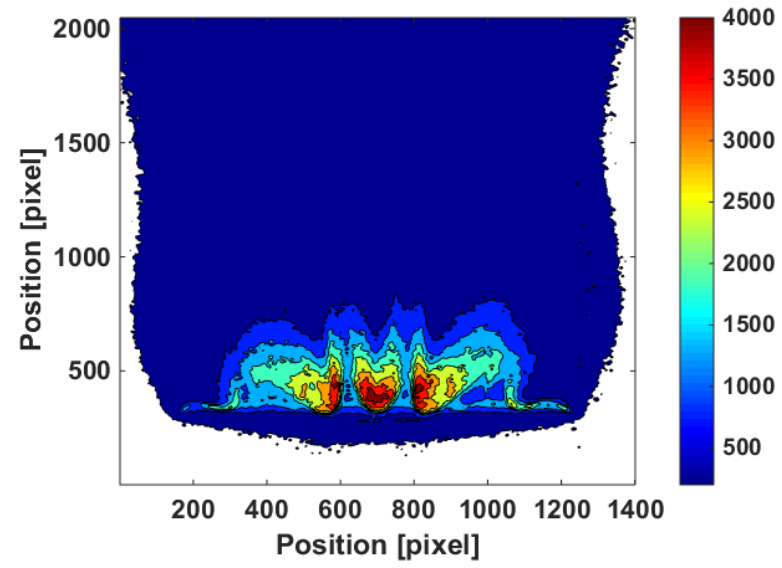

Fig. 14. C 10880-03, $60 \%$ gain, exposurer time $300 \mu \mathrm{s}$, contours of median smoothed image

\section{Results}

Data was acquired for exposure time: $20 \mu \mathrm{s}, 50 \mu \mathrm{s}, 300 \mu \mathrm{s}$ and image intensifier gain: $25 \%, 50 \%$. The last gain values were for both image intensifiers different. It was $75 \%$ for $\mathrm{C} 9547-03$ and $60 \%$ for $\mathrm{C} 10880-03$. This was due to the fact that the second amplifier was with $75 \%$ gain overexposed and the experiment could not be repeated. Exposure times for CCD camera were set-on $5000 \mu \mathrm{s}, 10000 \mu \mathrm{s}$ and $30000 \mu \mathrm{s}$. The results of the measurements are processed into the line charts (Fig.15,16,17 and 18) showing dependences of the image gradient and signal-to-noise ratio on the CCD camera exposure. For most modes, image gradient is increasing with longer exposure time of CCD camera. This effect is visible in characteristics with shorter image intensifier exposure time. Longer image intensifier exposure time can cause overwriting signal on phosphor screen and acquired data can be "smudged". This is the best visible at the picture Fig. 17. At the picture Fig. 16 is possible to see increasing, or constant characteristics, which is advantageous for better data obtaining by mere CCD exposure time increasing. This effect is possible to observe only in C9547-03 characteristics.

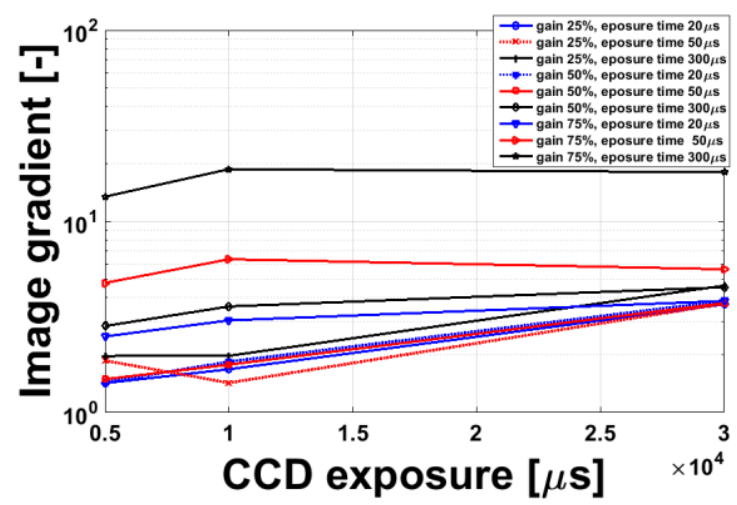

Fig. 15. Image gradient depending on CCD exposure time for different setup. Hamamatsu C9547-03

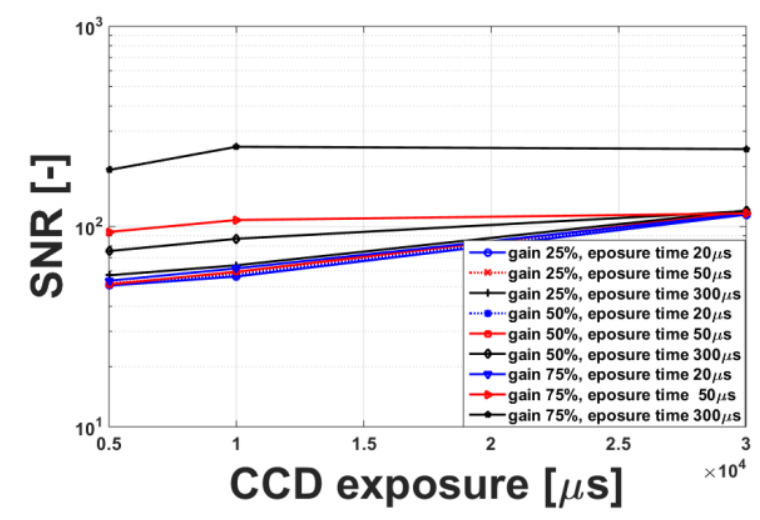

Fig. 16. Signal-to-noise ratio depending on $\mathrm{CCD}$ exposure time for different setup. Hamamatsu C9547-03

A C10880-03 characteristic does not shows improvement of observed image gradient and signal-to noise ratio after $10000 \mu$ s exposure time of CCD camera.

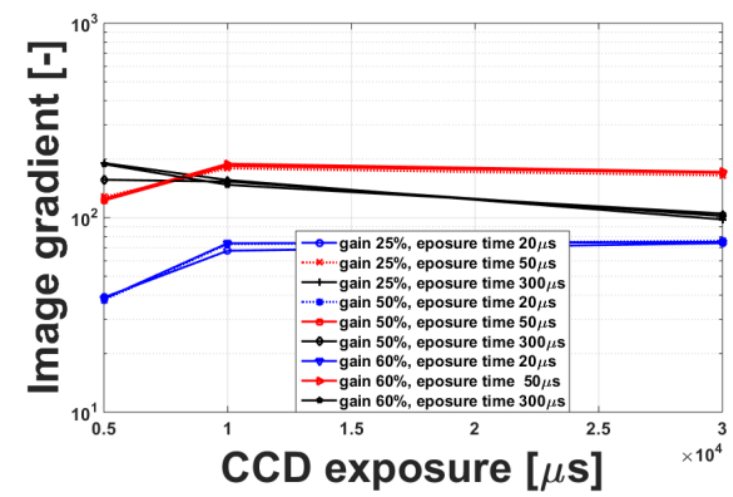

Fig. 17. Image gradient depending on $C C D$ exposure time for different setup. Hamamatsu C10880-03 
Image gradient for modes with shorter exposure time of image intensifier increases, and after mentioned value of CCD exposure time is the lines character unchanged. Course of signal-to-noise ratio increases only for the shortest image intensifier exposure time mode. The rest of courses decrease with longer exposure time of CCD.

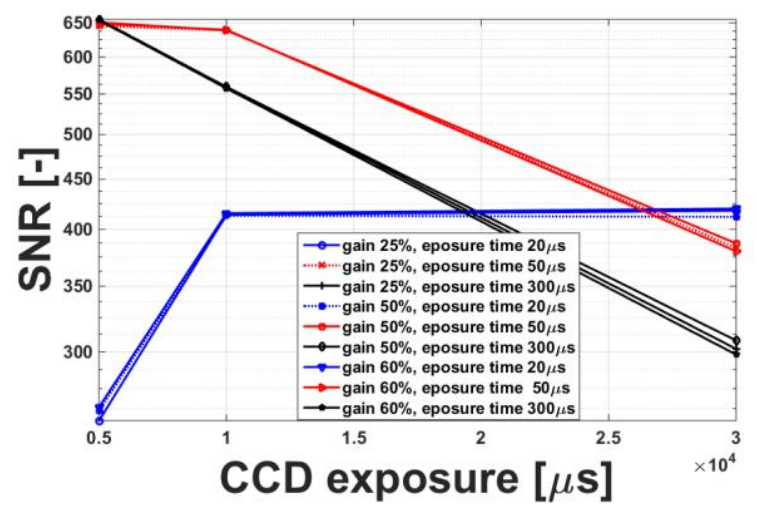

Fig. 18. Signal-to-noise ratio depending on $\mathrm{CCD}$ exposure time for different setup. Hamamatsu C10880-03.

\section{Conclusion}

This work aimed to verify the dependence of the synchronization of the recording equipment used for LIF and chemiluminescence methods. The results show that the quality of the recorded data is more affected by camera exposure time. On the other hand, the exposure time of the image intensifier increases the signal level, however, with the rising exposure time of the image intensifier the noise component also increases and the value of maximum image function gradient decreases and thus the detectability of the front flame position. The experiment has shown that with suitable synchronization the result with an increase of a signal-to-noise ratio by $38 \%$ for C10880-03 with screen P46 and $130 \%$ for C9547-03 with screen P43 can be obtained. The values of the image function gradient show increase by $55 \%$ for C10880-03 and 130\% for C9547-03, which can be significantly reflected in the capability of edge detection and therefore of the flame front. The performed experiment indicates that the image intensifier with a screen of P43 can use longer decay time of luminophore to signal collection and it can better data obtained. Image intensifier C10880-03 with screen P46 does not have mentioned effect, but it has stronger input signal then C9547-03. However this fact can be caused by different age and degradation of phosphor and microchannel plate inside C9547-03

\section{Acknowledgement}

This research has been realized using the support of The Ministry of Education, Youth and Sports program NPU I (LO), project \# LO1311 Development of Vehicle Centre of Sustainable Mobility. And support of EU Regional Development Fund in OP R\&D for Innovations (OP VaVpI) and The Ministry of Education, Youth and Sports, Czech Republic, project \# CZ.1.05/2.1.00/03.0125 Acquisition of
Technology for Vehicle Center of Sustainable Mobility This support is gratefully acknowledged

\section{References}

1. C. Crua, Combustion processes in a diesel engine Doctoral thesis, University of Brighton. (2002)

2. R.Demory, Optical Measurement of Nitric Oxide and Hydroxyl Radicals Distributions in Combusting Diesel Sprays, Doctoral thesis, University of Brighton. (2007)

3. F. Grich, M. Orain, Role of Planar Laser-Induced Fluorescence in Combustion Research, Aerospace Lab Journal, (11/2009)

4. V.Hlaváč, M. Sedláček, Zpracování signálu a obrazů. Vyd. 3. V Praze: ČVUT, 2009, vii 252s. ISBN 9788001044421

5. M. Jedlička, Zesilovače jasu obrazu. Světlo. Praha: FCC Public s. r. o., 2006, (04). ISSN 1212-0812

6. A.A. Verbeek, et al. Improved flame front curvature measurement for noisy OH-LIF images, University of Twente,Enshede (2013)

7. HAMAMATSU PHOTONICS K.K. High-speed gated image intensifier unit. Iwata, 2014. 